\title{
Image Interpolation Using 5/3 Lifting Scheme Approach
}

\author{
P.Ashok Babu ${ }^{1}$ and Dr.K.V.S.V.R Prasad ${ }^{2}$ \\ ${ }^{1}$ Department of Electronics \& Communication Engineering, NREC, Dhullapally, \\ Kompally, Hyderabad, A.P(state), INDIA. \\ ashokbabup2 @gmail. com. \\ ${ }^{2}$ D.M.S.S.V.H. College of Engineering, Machilipatnam, Krishna (Dt), A.P(state), \\ INDIA. \\ KVSVR@YAHOO. COM.
}

Abstract

In this paper we proposed fast and accurate interpolation and resizing of images using lifting scheme approach. 5/3 lifting scheme is an accurate and computationally inexpensive interpolation technique for image resizing. We compared the bilinear interpolation, Haar lifting scheme and 5/3 lifting scheme in this paper. The lifting scheme algorithm is applied for image interpolation to resize the image. In case of reduction in size, the image components are reduced and the reconstruction will be carried out to the original image. The reconstruction results are better by using Mean Squared Error (MSE) and Peak Signal to Noise Ratio (PSNR) with other techniques like bilinear interpolation and Haar lifting schemes. The interpolation and reconstruction is executed in much less time with better MSE and PSNR as compared to Bilinear and Haar lifting schemes.

\section{Keywords}

Lifting scheme, 5/3 Lifting transform, DWT, IDWT, Image Resizing, Reconstruction.

\section{INTRODUCTION}

Wavelets provide accurate and excellent enough tool to approximate the functions, datasets and signals. Mother wavelet acts as basic building block to approximate the complex signals and functions at reasonable computational overhead. In other words, wavelets can approximate complex signals by using a small set of coefficients without much computational complexity. This is possible because, most data sets have correlation both frequency domain and in time (or space) domain. Among the family of xxx let (wavelet, curvelet, ridgelet) transforms, wavelet is the first one to preserve the correlation in both frequency domain and spatial domain. Transformations in most of the frequency domain transforms do not preserve the original form of a signal like image in spatial domain. One cannot make out the visible form of an image from the results of cosine, sine or Discrete Fourier Transforms. However, the LPLP component of DWT of an image seems to be similar to the original image and other components are the edges in different directions. Thus DWT may serve as an excellent tool to simulate human vision phenomenon like continuous interpolation, because of the time-frequency localization property of wavelets.

\section{DISCRETE WAVELET TRANSFORM (DWT)}

When digital images are to be viewed or processed at multiple resolutions, the most popular mathematical tool is discrete wavelet transform [1]. Wavelet transform is the first transform which has been explored a lot in image and signal processing for its unique property of maintaining the spatial domain and frequency domain contents. Wavelet series expansion maps a 
function of continuous variables into a sequence of coefficients. Discrete wavelet transform decomposes the input image (2-D signal) into four different wavelet coefficients at the first level. The process of obtaining these four coefficients (Vetterli and Kovacevic, 1995) is presented in Fig.1. After dividing the data set into two parts that is even and odd, average and difference is computed. Out of these two coefficients, one is called the average and the other is the difference. These two coefficients are again processed to compute average-average, average-difference, difference-average and difference-difference terms. Thus after completion of the whole process we have four coefficients. The coefficient average-average is also known as LPLP coefficient (this is exactly visually similar to the original image but 50\% in size), average-difference as LPHP coefficient, difference-average as HPLP and

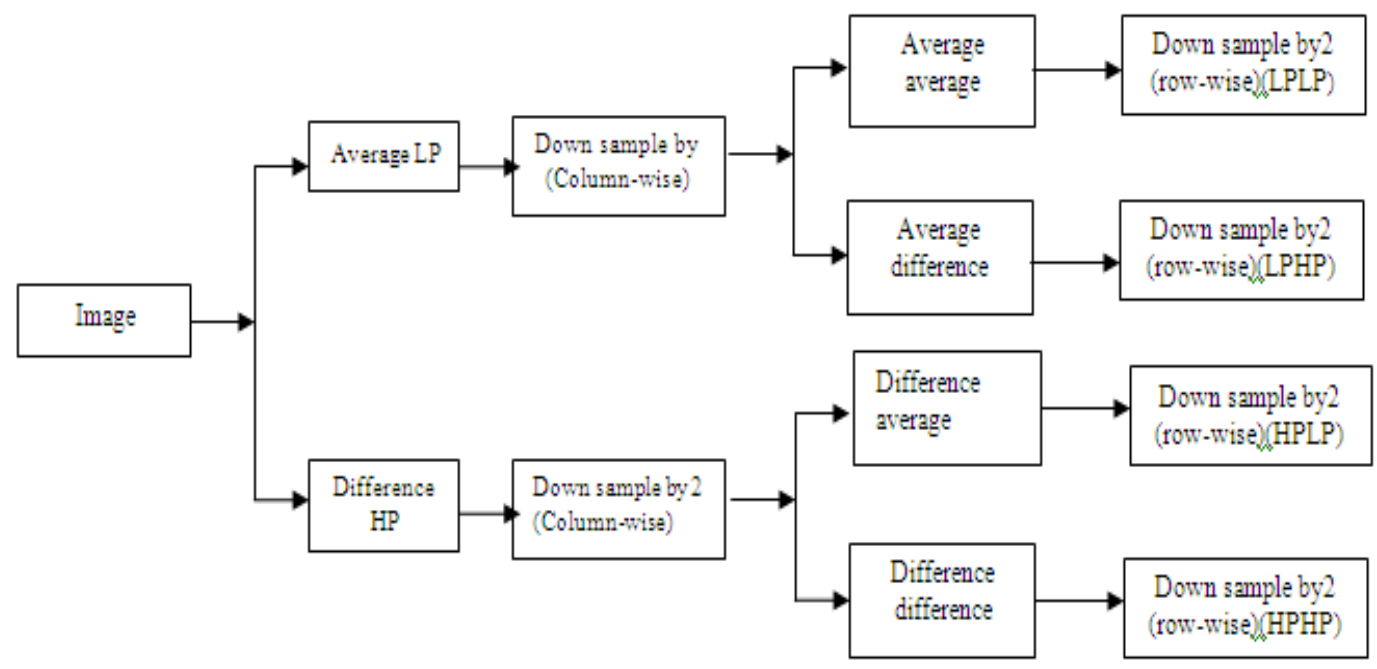

Fig. 1: QMF implementation of DWT

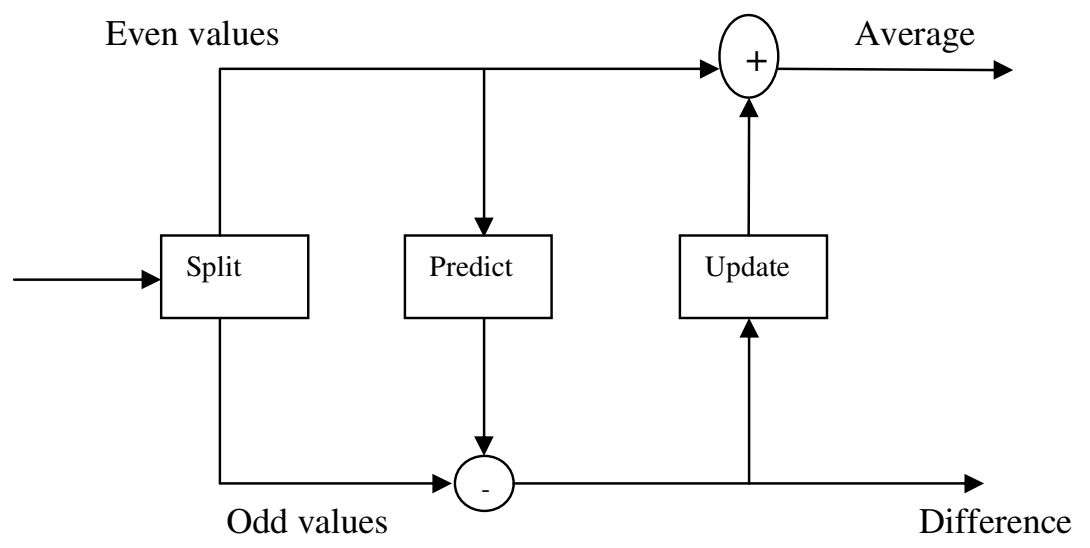

Fig. 2: Lifting scheme forward transform

difference-difference as HPHP. These four coefficients are exactly 50\% of the original image contain the different information of the original image as given below [2]. 
1).LPLP: This coefficient contains the low frequency image content and is the reduced resolution

2).LHLP: It contains Horizontal information of the image

3).HPLP: It contains vertical information of the image

4).HPHP: It contains diagonal information of the Image

\section{LIFTING SCHEME}

It is a technique to design wavelets and perform the discrete wavelet transform. The forward lifting scheme wavelet transform divides the data set being processed into an even half and an odd half [3].

\subsection{Lifting scheme forward transform}

Lifting scheme forward transform consists of three steps:

- Split

- Predict

- Update

3.1.1. Split: In split step the data is divided into ODD and EVEN elements.

3.1.2. Predict step: The difference between odd and even data forms the odd elements of the next step wavelet transformation. The predict step, where the odd value of next iteration is "predicted" from the even value of present step is described by (1). Index ' $j$ ' represents iteration and ' $i$ ' represents element:

$\operatorname{Odd}_{\mathrm{j}+1, \mathrm{j}}=\operatorname{odd}_{\mathrm{j}, \mathrm{i}}+\mathrm{P}\left(\mathrm{even}_{\mathrm{j}, \mathrm{i}}\right)$

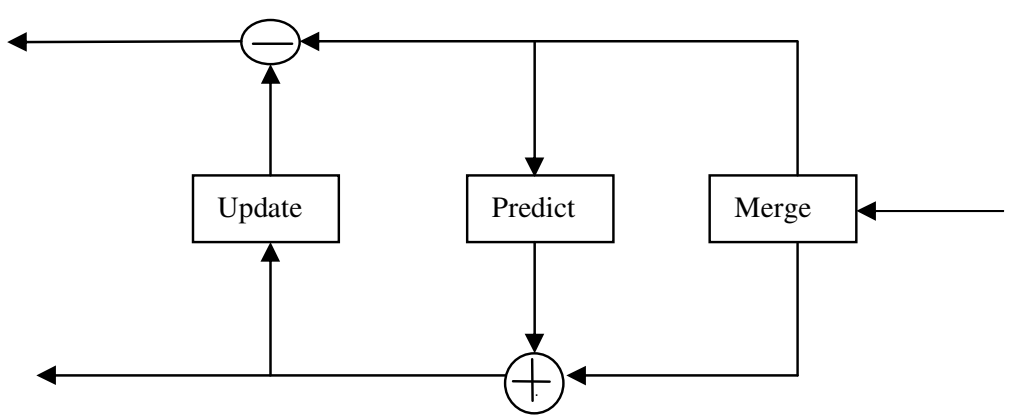

Fig. 3: Lifting scheme inverse transform

3.1.3. Update step: The update step replaces the even elements of the next step with average of the earlier step. These results in a smoother input (even element) for the next step wavelet transform. The update step follows the predict phase. So in calculating average the update phase must operate on the differences that are stored in the odd elements:

Even $_{\mathrm{j}+1, \mathrm{i}}=$ even $_{\mathrm{i}, \mathrm{j}}+\mathrm{U}\left(\operatorname{odd}_{\mathrm{j}+1, \mathrm{i}}\right)$

A simple lifting scheme forward transform is as shown in Fig. 2. 
After dividing the complete data set into two parts that is even and odd, the processing is done as follows: For the forward transform iteration $\mathrm{j}$ and element $\mathrm{i}$, the new odd element $\mathrm{j}+1, \mathrm{i}$ would be: $\operatorname{Odd}_{\mathrm{j}+1, \mathrm{i}}=$ odd $_{\mathrm{j}, \mathrm{j}}-\mathrm{even}_{\mathrm{j}, \mathrm{i}}$

Even element of the next step is calculated as: $\operatorname{Even}_{\mathrm{j}+1, \mathrm{i}}=\left(\operatorname{even}_{\mathrm{j}, \mathrm{i}}+\operatorname{odd}_{\mathrm{j}, \mathrm{i}}\right) / 2$

The original value of the odd $j$, $i$ element has been replaced by the difference between this element and its even predecessor. Simple algebra lets us recover the original value from Eq. 3:

$\operatorname{Odd}_{\mathrm{j}, \mathrm{i}}=\operatorname{even}_{\mathrm{j}, \mathrm{i}}$ odd $_{\mathrm{j}+1, \mathrm{I}}$

Substituting this into the average that is Eq. 4

$\operatorname{Even}_{\mathrm{j}+1, \mathrm{i}}=\left(\operatorname{even}_{\mathrm{j}, \mathrm{i}}+\operatorname{even}_{\mathrm{j}, \mathrm{i}}+\operatorname{odd}_{\mathrm{j}+1, \mathrm{i}}\right) / 2$

$\operatorname{Even}_{\mathrm{j}+1, \mathrm{i}}=\operatorname{even}_{\mathrm{j}, \mathrm{i}}+\left(\operatorname{odd}_{\mathrm{j}+1, \mathrm{i}}\right) / 2$

\subsection{Lifting scheme inverse transform}

As the name suggests the mirror process of forward lifting scheme transform. We recover the original data sequence by going upwards as shown in Fig. 3. Additions are substituted for subtractions and subtractions for additions. The merge step replaces the split step.

\section{MATERIALS AND METHODS}

\subsection{Image resizing using lifting scheme}

Image is a data set made of rows and columns. Monochrome images are two dimensional with one intensity value associated with every pixel [4]. Being two dimensional, they are to be processed first row wise and then column wise assuming that the image signal is orthogonal in both the directions. Here, we present computation of one dimensional wavelet transform using lifting scheme and then extend it to the 2D images to compute the Haar Wavelet transform. Consider the following pixel row intensity data array:

$\mathrm{S}=\{44,20,30,48,49,39,40,35\}$

Step 1 (split): Initially this data set is divided into two parts. First, third, fifth, seventh pixel are accommodated in the first part known as odd part. Second, forth, sixth, eighth pixel are accommodated in the second part known as even part that is:

Odd $=44,30,49,40$ and Even $=20,48,39,35$

Now this even and odd data parts are processed in the following stepwise manner to obtain average and difference terms.

Step 2 (predict): The first difference is obtained by subtracting the first even element from first odd element. Similar procedure is adopted for remaining all elements of odd and even vector sequentially, to result in even ${ }_{j+1, i}$ and here DIFF1 vector sequence as given below:

even $_{\mathrm{j}+1, \mathrm{i}}=\operatorname{odd}_{\mathrm{j}+1, \mathrm{i}}-$ even $_{\mathrm{j}, \mathrm{i}}$ and thus

DIFF1 $=\{44,-18,10,5\}$. 
Step 3 (update): The first average term is obtained by adding the first odd and first even element and by dividing this sum by two. This process is continued for the complete data set of odd and even elements sequentially and the resultant vector odd ${ }_{j+1, i}$ and AVG1 is obtained as shown below:

Even $_{\mathrm{j}+1, \mathrm{i}}=\left(\right.$ even $\left._{\mathrm{j}, \mathrm{i}}+\operatorname{odd}_{\mathrm{j}, \mathrm{i}}\right) / 2$ thus

AVG1 $=\{32,39,44$, and 37.5$\}$.

Thus initial eight elements present in a data array are reduced to four average and four difference terms. Same procedure from Step 1-3 is repeated further, considering AVG1 vector as input data array for the next level forward lifting scheme transformation. Thus the successive transformations can be taken till each component reaches single coefficient.

For image processing applications, this procedure of Step 1-3 is applied to all the rows present in the image. The resulting matrix is subjected to the same procedure of 1-3 Steps on all columns in the image. This results in Low Pass Low Pass (LPLP) approximation of the image and is the 50\% reduced version of the original image. Thus at each level, the LPLP (AVG component after rowwise and column-wise computation of lifting scheme DWT) is considered as the interpolated version of the original image. The difference components obtained at every stage are used for reconstruction of the original image. For the next level lifting scheme forward transformation average component of earlier level acts as an input. At every lifting scheme forward transformation, image size gets reduced to $1 / 2$ of the input image size. Thus finally individual the component (LPLP, LPHP, HPLP and HPHP) of an image gets reduced to single DWT coefficient after successive transformations[5].

\subsection{Reconstruction of the image using Inverse discrete wavelet transforms}

The original data can be reconstructed with the help of inverse lifting scheme. Four pixels obtained in forward lifting scheme transform now acts as an input to reconstruct average vector in, inverse lifting scheme. Proceeding further in this manner as explained in materials and methods till all average vectors (obtained in various levels of forward lifting scheme transformation) gets processed, yields all the recovered elements in the last step. These reconstructed elements correspond to the respective elements in the original array. Similar procedure is adopted in row wise and column wise manner for the image. Image resizing with the algorithm as presented in this paper is not possible if the image is not available in the size $2^{\mathrm{n}} \times 2^{\mathrm{n}}$. The size of the original image with rows ' $r$ ' and columns ' $c$ ' is converted into the next $\left(2^{\mathrm{n}} \times 2^{\mathrm{n}}\right)$ size by zero padding at the ends of all rows and columns i.e., adding $\left(2^{n}-r\right)$ zeros in all the columns and $\left(2^{\mathrm{n}}-\mathrm{c}\right)$ zeros in all the rows at the ends.

\section{5/3 LIFTING TRANSFORM}

A wavelet $5 / 3$ lifting transform has 3 and 5 taps in the high and low pass analysis filters respectively. 5/3 lifting transform is also known as Le Gall 5/3 transform. Le Gall 5/3 wavelet is the shortest symmetrical bi-orthogonal wavelet with two vanishing moments. It is the simplest way to decompose the image into one high frequency component and one low frequency component. The shortest bi orthogonal scaling and wavelet function with two regularity factors at synthesis and analysis denoted $(2,2)$ is attained with Le Gall $5 / 3$ synthesis function. Interpolation of data means inserting additional data points into the sequence to increase the sampling rate. 
Let assume the pixels $\mathrm{x}_{1}, \mathrm{x}_{2}, \mathrm{x}_{3}, \mathrm{x}_{4}, \mathrm{x}_{5}, \mathrm{x}_{6} \ldots$ and in general we will predict even pixels and update the odd pixels. The formulas used to predict and update even and odd pixels using 5/3 lifting are

$\mathrm{P}_{\mathrm{n}}=\mathrm{x}_{4}-\left(\left(\mathrm{x}_{3}+\mathrm{x}_{5}\right) / 2\right)$

Where $P_{n}$ is the prediction of even ' $n$ ' pixels

$\mathrm{U}_{\mathrm{n}}=\mathrm{x}_{3}+\left(\left(\mathrm{p}_{2}+\mathrm{p}_{4}\right) / 4\right)$

Where $\mathrm{U}_{\mathrm{n}}$ is updation of odd pixels

\subsection{5/3 Lifting Algorithm}

1) Read the original image

2) For each scaling factor read the reference image and perform $5 / 3$ lifting to predict and update the image along horizontal and vertical directions.

3) For each down scaling level, down sample the update coefficients by a factor of two and perform $5 / 3$ lifting as above step.

4) For each up scaling perform nearest neighbour interpolation on pred samples and apply inverse $5 / 3$ lifting.

\section{RESULTS AND DISCUSSION}

From the results we can conclude that $5 / 3$ lifting scheme will give more efficient results compared to Bilinear and Haar lifting schemes. Fig.5 and Fig.7 are the comparisons of Mean Square Error (MSE) and Peak Signal to Noise Ratio (PSNR). Fig.4 and Fig.6 are the resizing images using 5/3 lifting with different resize factors for Lena and Zelda. The proposed algorithm is mainly focused on MSE and PSNR values. If we compare the three algorithm the $5 / 3$ lifting scheme will give the better results compared to bilinear and Haar lifting schemes.

From the table 1 we compared the MSE and PSNR values for Lena image with different resizing factors. From the table 2 we compared the MSE and PSNR values for Zelda image. From the two tables we can observe that the 5/3 lifting scheme will gives the better PSNR values and low MSE values than the Haar lifting scheme and bilinear interpolation. If we increase the resize factor for an image the PSNR value increases and the MSE value decreases. If we consider large resize factor the time taken by the image to resize increases and the efficiency of the algorithm may decreases. The lifting scheme allows a faster implementation of the wavelet transform. Traditionally, the fast wavelet transform is calculated with a two-band sub band transform scheme, in Fig. 1. In each step the signal is split into a high pass and low pass band and then sub sampled. Recursion occurs on the low pass band. The lifting scheme makes optimal use of similarities between the high and low pass filters to speed up the calculation.

\section{CONCLUSION}

In this particular paper we used $2^{\mathrm{n}} \times 2^{\mathrm{n}}$ images for resizing. The proposed algorithm will resize the image if the image is of the size $2^{\mathrm{n}} \times 2^{\mathrm{n}}$ and zero padding enables application of the scheme on all sizes of images but with additional computational overhead. If we use 
the large images for resizing it will take more time to resize. So we can concentrate on these large images as a future work to resize the images and to increase the more efficiency.

Table1:Comparision of MSE and PSNR values for Lena Image

\begin{tabular}{|c|c|c|c|c|c|c|c|c|}
\hline \multicolumn{2}{|c|}{} & \multicolumn{2}{c|}{$\begin{array}{c}\text { Bilinear } \\
\text { Interpolation }\end{array}$} & \multicolumn{2}{c|}{ Haar Lifting } & \multicolumn{2}{c|}{$5 / 3$ Lifting } \\
\hline Image & Size & $\begin{array}{c}\text { Resize } \\
\text { Factor }\end{array}$ & MSE & PSNR & MSE & PSNR & MSE & PSNR \\
\hline Lena & $\begin{array}{c}128 \times \\
128\end{array}$ & 0.25 & 866.8743 & 18.7512 & 492.0217 & 21.2110 & 332.8713 & 22.9080 \\
\hline Lena & $\begin{array}{c}256 \times \\
256\end{array}$ & 0.5 & 520.6248 & 20.9656 & 164.7891 & 25.9615 & 87.7668 & 28.6975 \\
\hline Lena & $\begin{array}{c}1024 \times \\
1024\end{array}$ & 2 & 188.9562 & 25.3672 & 82.6570 & 28.9580 & 38.8632 & 32.2354 \\
\hline Lena & $\begin{array}{c}2048 \times \\
2048\end{array}$ & 4 & 159.0445 & 26.1156 & 124.2400 & 27.1882 & 77.5673 & 29.2340 \\
\hline
\end{tabular}

Table2:Comparision of MSE and PSNR values for Zelda Image

\begin{tabular}{|c|c|c|c|c|c|c|c|c|}
\hline \multicolumn{2}{|c|}{} & \multicolumn{2}{c|}{$\begin{array}{c}\text { Bilinear } \\
\text { Interpolation }\end{array}$} & \multicolumn{2}{c|}{ Haar Lifting } & \multicolumn{2}{c|}{$5 / 3$ Lifting } \\
\hline Image & Size & $\begin{array}{c}\text { Resize } \\
\text { Factor }\end{array}$ & MSE & PSNR & MSE & PSNR & MSE & PSNR \\
\hline Zelda & $\begin{array}{c}128 \times \\
128\end{array}$ & 0.25 & 409.8525 & 22.0045 & 176.6397 & 25.6599 & 96.9163 & 28.2668 \\
\hline Zelda & $\begin{array}{c}256 \times \\
256\end{array}$ & 0.5 & 210.2132 & 24.9042 & 46.1388 & 31.4901 & 21.8254 & 34.7412 \\
\hline Zelda & $\begin{array}{c}1024 \times \\
1024\end{array}$ & 2 & 87.2603 & 28.7226 & 26.9042 & 33.8326 & 12.7555 & 37.0738 \\
\hline Zelda & $\begin{array}{c}2048 \times \\
2048\end{array}$ & 4 & 74.9172 & 29.3850 & 41.3637 & 31.9646 & 25.5541 & 34.0562 \\
\hline
\end{tabular}

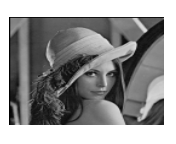

$4(\mathrm{a})$

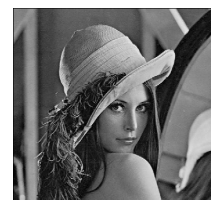

4. (c)

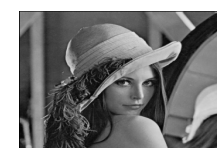

4.(b

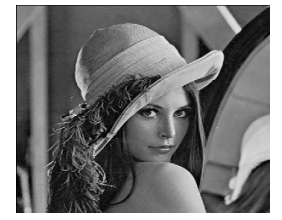

4. (d)

Fig.4: 5/3 Lifting based Image Resizing with resizing factors of (a) : 0.25 , (b) :0.5, (c) : 2, (d) : 4, For Lena 
Signal \& Image Processing : An International Journal(SIPIJ) Vol.2, No.1, March 2011

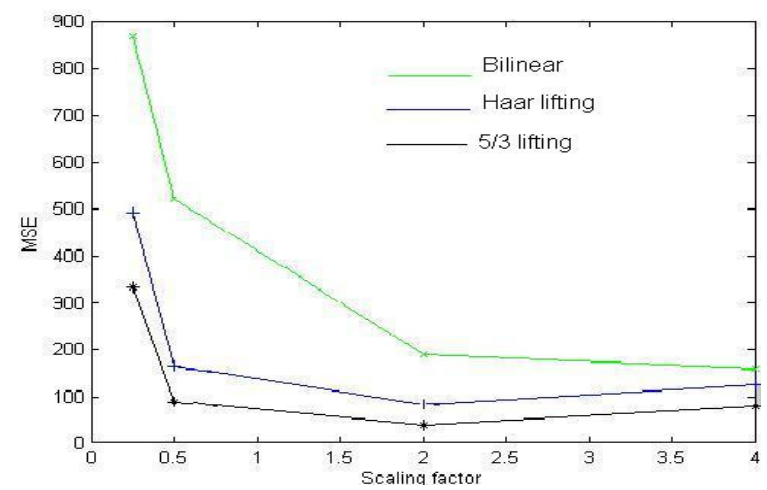

5(a)

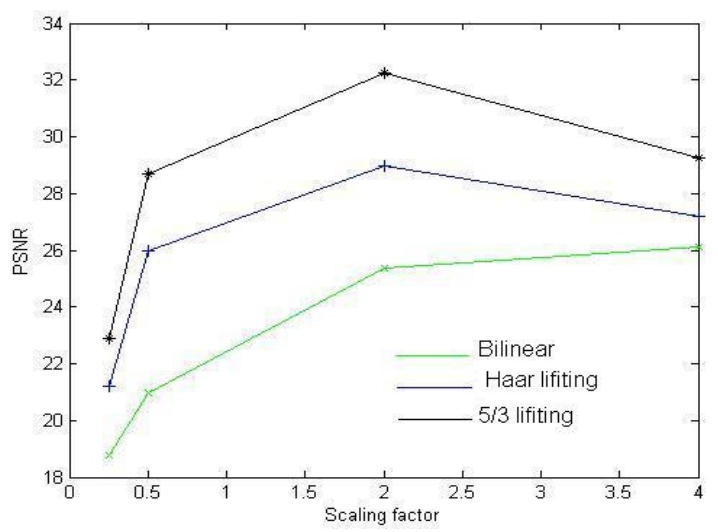

5(b)

Fig. 5: (a) Comparison of MSE and (b) Comparison of PSNR for Lena Image.
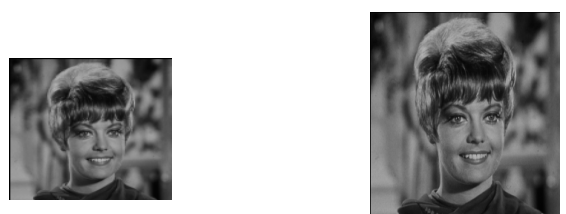

6. (a)

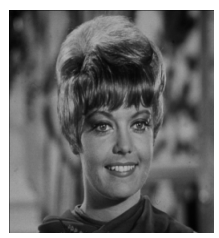

6.(c)
6. (b)

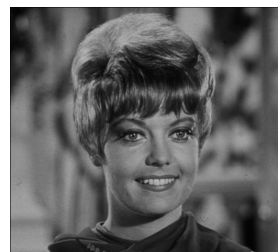

6.(d)

Fig.6: 5/3 Lifting based Image Resizing with resizing factors of (a) : 0.25, (b) :0.5, (c) : 2, (d) : 4 for Zelda 
Signal \& Image Processing : An International Journal(SIPIJ) Vol.2, No.1, March 2011

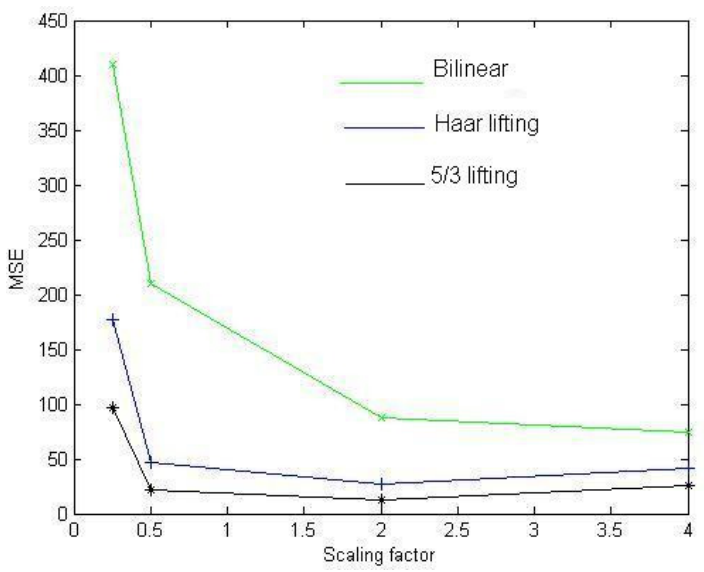

7(a)

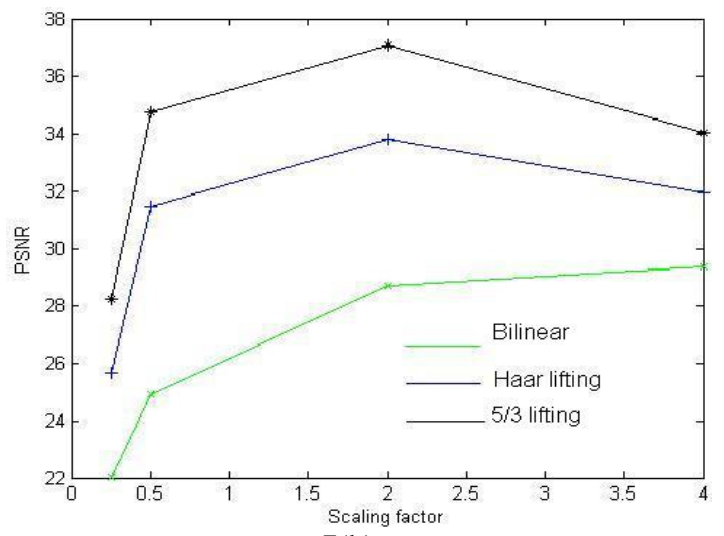

7(b)

Fig. 7: (a) Comparison of MSE and (b) Comparison of PSNR for Zelda Image. 
Signal \& Image Processing : An International Journal(SIPIJ) Vol.2, No.1, March 2011

\section{REFERENCES}

[1]. Asamwar, R.S., K.M. Bhurchandi and A.S.Gandhi, 2009. "Piecewise lifting scheme based DWT to model human vision interpolation phenomenon”. Proceeding of the IAENG International Conference on Signal and Image Engineering, July 1-3, IAENG, London, UK., pp: 822-827. http://www.iaeng.org/publication/WCE2009/WCE 2009_pp822-827.pdf

[2]. Asamwar, R.S., K.M. Bhurchandi and A.S. Gandhi, 2010. "Interpolation of images using discrete wavelet transform to simulate image resizing as in human vision”. Int. J. Autom. Comput., 7: 9-16. DOI: $10.1007 / \mathrm{S} 116333-010-0009-$

[3]. Daubechies, I. And W. Sweldens, 1998. "Factoring wavelet transforms into lifting steps". J. Fourier Anal. Appli., 49: 247-269. DOI: 10.1.1.106.8124

[4] Latha, Y.M., B.C. Jinaga and V.S.K. Reddy, 2007. “A precise content based image retrieval: Lifting Scheme”. Int. J. Comput. Sci. Network Secur. 7: 38-45.

[5] Asamwar, R.S., K.M. Bhurchandi and A.S. Gandhi, 2010. "Successive Image Interpolation Using Lifting Scheme” Approach. Journal of Computer Science 6 (9): 961-970

${ }^{1}$ Mr.P.Ashok Babu obtained B.E Degree in 2001 from Andhra University, M.E Communication Engineering) in 2005 Osmania University. He pursuing the Ph.D. from JNTUH university, Hyderabad in Digital Image Processing. He published two papers in International journals and one paper in International Conference. He is member of IACSIT and IAENG . He worked in various positions in the Department of ECE, GRIET, Hyderabad. Presently he is working as Assoc. Professor and Head, Department of ECE, Narasimha Reddy Engineering College, Dhullapally, Hyderabad, Andhra Pradesh (state) India.

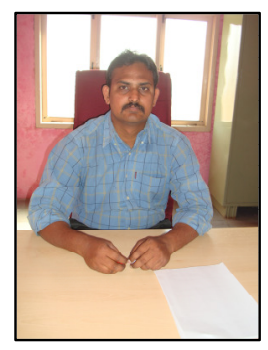

${ }^{2}$ Dr. K.V.S.V.R Prasad obtained B Sc. Degree in 1963 from Andhra University, B.E (Telecommunication Engineering) in 1967 and M.E (ECE) Microwave Engineering specialization in 1977 from Osmania University. He received the Ph.D. in 1985 from IIT Kharagpur in strip and micro strip transmission lines. He published six papers in IEEE Transactions in MTT, Antenna and Propagation and EMI/EMC and three papers in National Conferences. He is fellow of IETE (life member). He worked in various capacities in the Department of ECE, Osmania University, Hyderabad. Presently he is working as professor and head, Department of ECE, D.M.S.S.V.H. college of engineering, Machilipatnam, Andhra Pradesh (state) India.

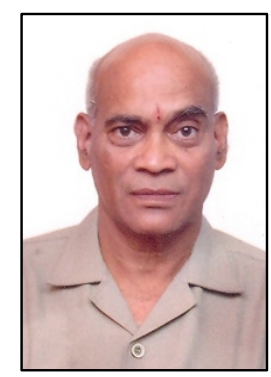

